(2) Open Access Full Text Article

\title{
In vitro parallel evaluation of antibacterial activity and cytotoxicity of commercially available silver- containing wound dressings
}

This article was published in the following Dove Press journal:

Chronic Wound Care Management and Research

7 January 2015

Number of times this article has been viewed

\author{
Shunji Yunoki \\ Masushi Kohta ${ }^{2}$ \\ Yoshimi Ohyabu' \\ Tetsuji Iwasaki \\ 'Biotechnology Group, Tokyo \\ Metropolitan Industrial Technology \\ Research Institute, ${ }^{2}$ Medical Engineering \\ Laboratory, ALCARECo., Ltd, Tokyo, \\ Japan
}

Correspondence: Shunji Yunoki Biotechnology Group, Tokyo Metropolitan Industrial Technology Research Institute, 2-4-I0 Aomi, Koto-ku, Tokyo 135-0064, Japan

Tel +8I 35530267 |

Fax +81355302627

Email yunoki.shunji@iri-tokyo.jp
Purpose: This study evaluated the in vitro antibacterial activity and cytotoxicity of various commercially available silver-containing dressings (Ag dressing).

Methods: Biohesive Ag (hydrocolloid, silver sulfadiazine), Aquacel ${ }^{\circledR} \mathrm{Ag}$ (nonwoven fabric, ionic silver $\left[\mathrm{Ag}^{+}\right]$), Algisite ${ }^{\mathrm{TM}} \mathrm{Ag}$ (nonwoven fabric, $\mathrm{Ag}^{+}$), Mepilex ${ }^{\circledR} \mathrm{Ag}$ (foam, silver sulfate), and PolyMem ${ }^{\circledR} \mathrm{Ag}$ (foam, nanocrystalline silver) were tested for characteristics of $\mathrm{Ag}^{+}$release, antibacterial activity, and cytotoxicity. The release of $\mathrm{Ag}^{+}$was investigated in cell culture medium at immersion periods of 6, 24, and 48 hours. The antibacterial activity against Staphylococcus aureus and Pseudomonas aeruginosa were accessed by a disc diffusion test. The cytotoxicity was evaluated using V79 cells, by an extraction method.

Results: The cytotoxicity was not a monotonic function of the antibacterial activity among the $\mathrm{Ag}$ dressings and could not be simply explained by $\mathrm{Ag}^{+}$-release properties. Biohesive $\mathrm{Ag}$ was regarded as a slow-release Ag dressing, showing the lowest cytotoxicity, while the antibacterial activity was classified as "strong" or "significant" against the two species of bacteria. Aquacel Ag and Algisite Ag showed higher antibacterial activity and cytotoxic effects, which were supported by the higher $\mathrm{Ag}^{+}$release. Mepilex $\mathrm{Ag}$ showed the highest release of $\mathrm{Ag}^{+}$, and the cytotoxicity was the highest among the Ag dressings. However, the antibacterial activity was classified as "significant" or "no activity" for $P$. aeruginosa and $S$. aureus, respectively. PolyMem Ag showed the lowest $\mathrm{Ag}^{+}$release, and the antibacterial activity classified as "significant" or "no activity" for $S$. aureus and P. aeruginosa, respectively, whereas the cytotoxicity was similar to those of Aquacel Ag and Algisite Ag.

Conclusion: The efficacy and adverse effects of the Ag dressings revealed differences that should be considered by clinicians during wound management.

Keywords: ionic silver, silver sulfadiazine, nanocrystalline silver, wound management, wound infection, slow release

\section{Introduction}

Several types of silver-containing wound dressing (Ag dressing) are commercially available and clinically used for standard wound care. ${ }^{1,2}$ Silver ion $\left(\mathrm{Ag}^{+}\right)$is the bioactive state of silver; it is formed when $\mathrm{Ag}^{+}$are displaced by cations in anionic polymer substrates ${ }^{3}$ or when silver compounds capable of releasing $\mathrm{Ag}^{+}$are incorporated into polymer substrates. ${ }^{4}$ Silver-containing dressings (Ag dressings) are used to reduce the risk of wound infection ${ }^{1,5}$ because of the broad antibacterial spectrum of silver. ${ }^{6-10} \mathrm{Ag}^{+}$reacts with membrane proteins and the DNA of bacteria, leading to denaturation of the proteins and interference in DNA replication. ${ }^{11,12}$ Clinical evidence on Ag dressings has accumulated from clinical trials on patients with intractable ulcers, ${ }^{13,14}$ resulting in an international 
consensus on the efficacy and cost effectiveness of $\mathrm{Ag}$ dressings, published by Wounds International in 2012. ${ }^{15}$

Despite the beneficial effect of silver, some adverse effects of topical silver on wound healing have also been described. ${ }^{16-18}$ Delayed wound healing has been explained by cytotoxic effects and histological damage resulting from excessive $\mathrm{Ag}^{+}$release to wound sites. ${ }^{19}$ Cytotoxic effects of silver have been demonstrated by in vitro and in vivo studies, in diabetic fibroblasts, ${ }^{18}$ human $\mathrm{HaCaT}$ keratinocytes,${ }^{20}$ and in tissue culture and animal models. ${ }^{21}$ Antibacterial activity and cytotoxicity are inherently in a trade-off relationship; therefore, the behavior of antibacterial silver in wound sites should be regulated, depending on the conditions of wounds, to achieve sufficient antibacterial activity while simultaneously minimizing cytotoxic effects.

The antibacterial effects of Ag dressings and their adverse effects may primarily depend on the characteristics of $\mathrm{Ag}^{+}$ release. ${ }^{22}$ The release of $\mathrm{Ag}^{+}$is determined by several factors, including electrostatic interaction of $\mathrm{Ag}^{+}$and anionic substrates, water solubility of the silver compounds, and the structure and hydrophilicity of substrates, which regulate water permeation and diffusion. Silver nitrate has the highest water solubility $\left(219 \mathrm{~g} / 100 \mathrm{~cm}^{3}\right)$ among inorganic silver compounds and is used for the preparation of $\mathrm{Ag}$ dressings, in which $\mathrm{Ag}^{+}$ is adsorbed to charged groups of polymer substrates. ${ }^{20}$ Silver sulfate, with lower water solubility $\left(0.8 \mathrm{~g} / 100 \mathrm{~cm}^{3}\right.$ at $\left.20^{\circ} \mathrm{C}\right)$, is also incorporated into dressings. A hydrocolloid dressing containing silver sulfadiazine (AgSD) has been recently released commercially. Before the release of this dressing, AgSD, with its extremely low water solubility $(3 \mu \mathrm{M}),{ }^{23}$ was widely used for topical antibacterial ointments. ${ }^{12} \mathrm{Ag}^{+}$or silver compounds are incorporated into dressings in different manners, suggesting that different characteristics of silver release are exhibited. Furthermore, various polymeric substrates are used, including foam, nonwoven fabrics, or hydrocolloids, which alter diffusion of $\mathrm{Ag}^{+}$. However, there have been few studies that evaluated the antibacterial activity and cytotoxic effects of various commercially available $\mathrm{Ag}$ dressings in parallel. ${ }^{20}$
The purpose of this study was to evaluate the antibacterial activity and cytotoxicity of various commercially available Ag dressings in parallel. We focused on the relationship between antibacterial activity and cytotoxic effects, which is believed to be useful information for clinicians addressing risk management for infected wounds. Five Ag dressings, composed of various silver compounds $\left(\mathrm{Ag}^{+}\right.$, silver sulfate, nanocrystalline silver, and AgSD) and substrates (nonwoven fabrics, foam, and hydrocolloid) were employed, and their antibacterial activity and cytotoxicity were evaluated in vitro. $\mathrm{Ag}^{+}$-release properties of the $\mathrm{Ag}$ dressings were also evaluated, to explain the functions of Ag dressings. The cytotoxicity was not a monotonic function of the antibacterial activity among Ag dressings and could not be simply explained by $\mathrm{Ag}^{+}$-release properties. Our findings suggested that Ag dressings have different characteristics depending, not only on $\mathrm{Ag}^{+}$release but also, other factors, such as species of silver released and substrate properties, and should be chosen according to wound conditions.

\section{Material and methods Ag dressings}

Five Ag dressings were employed. The product names of dressings, their manufacturers, and types of silver compounds and substrates are listed in Table 1. The product data state that all of these dressings can be applied to chronic wounds, such as pressure ulcers or diabetic foot ulcers.

\section{Silver release test}

The characteristics of silver release from the Ag dressings were assessed using minimum essential medium (MEM) containing serum or ultrapure water as the releasing medium. All specimens were cut to discs (diameter of $10 \mathrm{~mm}$; area of $0.785 \mathrm{~cm}^{2}$ ) and weighed. The specimens were immersed in $5 \mathrm{~mL}$ of the releasing medium at $37^{\circ} \mathrm{C}$. After an immersion period of 6 , 24 , or 48 hours $(n=3)$, the specimens were collected. The microresidues in the releasing media were removed with a membrane filter (pore size $0.45 \mu \mathrm{m}$ ). $\mathrm{Ag}^{+}$standard solutions

Table I Characteristics of $\mathrm{Ag}$ dressings employed in this study

\begin{tabular}{|c|c|c|c|c|}
\hline Product name & Manufacturer & Substrate & Silver compound & $\begin{array}{l}\text { Weight per } \\
\text { area }\left(\mathrm{mg} / \mathrm{cm}^{2}\right)\end{array}$ \\
\hline Biohesive Ag & ALCARE & Hydrocolloid & Silver sulfadiazine & $|3|$ \\
\hline Aquace ${ }^{\circledR} \mathrm{Ag}$ & ConvaTec & Nonwoven fabric & Ionic silver & 12.1 \\
\hline Algisite $^{\mathrm{TM}} \mathrm{Ag}$ & Smith and Nephew & Nonwoven fabric & lonic silver & 16.9 \\
\hline Mepilex ${ }^{\circledast} \mathrm{Ag}$ & Mölynlycke Health Care & Foam & Silver sulfate & 67.7 \\
\hline PolyMem ${ }^{\circledR} \mathrm{Ag}$ & Ferris MFG & Foam & Nanocrystalline silver & 78.7 \\
\hline
\end{tabular}

Note: Full manufacturer details are as follows: ALCARE, Tokyo, Japan; ConvaTec, Princeton, NJ, USA; Smith and Nephew, London, UK; Mölnlycke Health Care, Gothenburg, Sweden and Ferris MFG, Burr Ridge, IL, USA.

Abbreviation: Ag dressings, silver-containing dressings. 
(0-0.2 ppm) were prepared by dilution of silver inductively coupled plasma (ICP) standard (1,000 ppm in dilute nitric acid) (Merck KGaA, Darmstadt, Germany) with ultrapure water. The sample solutions were assayed with reagents for the $\mathrm{Ag}^{+}$-measuring apparatus (AGT-131; Nihon Ion Co., Tokyo, Japan). The assay system was based on a conventional method, using 4-(3,5-dibromo-2-pyridylazo)-N,N-dimethylaniline and sodium dodecyl sulfate for determining $\mathrm{Ag}^{+}$concentrations. ${ }^{24}$ The releasing media were diluted with ultrapure water to achieve $\mathrm{Ag}^{+}$concentrations $<0.2 \mathrm{ppm}$. The colored solutions were measured spectrophotometrically, and the optical density at $570 \mathrm{~nm}$ was used for determining $\mathrm{Ag}^{+}$concentrations.

\section{Antibacterial evaluation}

The antibacterial activity of the dressings was evaluated by the disc diffusion test, as described in the Japanese Industrial Standards number L 1902, ${ }^{25}$ which indicates the diffusion of antibacterial agents from specimens; we then counted the bacteria at the interface between the specimens and the agar gels for quantification of the antibacterial activity on the surface of specimens. The gram-positive bacterium Staphylococcus aureus (American Tissue Culture Collection [ATCC] 12783) and gramnegative Pseudomonas aeruginosa (ATCC 3080), commonly found in infected wounds, ${ }^{26}$ were used as test bacteria. The bacteria were precultured on agar plates containing Bacto ${ }^{\mathrm{TM}}$ Yeast Extract (BD Bioscience, Franklin Lakes, NJ, USA) and Polypeptone $^{\mathrm{TM}}$ (Nihon Pharmaceutical, Tokyo, Japan), and cultured under shaking (100 rpm) with liquefied medium, at $37^{\circ} \mathrm{C}$ for 4.5 hours. The bacterial suspension $\left(3.4 \times 10^{7}\right.$ colony forming unit (CFU)/mL for $S$. aureus; $5.4 \times 10^{6}$ for $P$. aeruginosa) was diluted ten times, and aliquots $(200 \mu \mathrm{L})$ were spread onto agar plates. The plates were subjected to the following tests.

\section{Disc diffusion test}

The disc specimen (diameter of $20 \mathrm{~mm}$ ) was placed on the center of the agar plate and then incubated at $37^{\circ} \mathrm{C}$ for 24 hours. If inhibitory concentrations were reached, a clear (inhibition) zone without colonies could be seen around the disc specimens. The width of the inhibition zone was measured.

\section{Counting of bacteria}

The agar plates used in the agar diffusion test were then tested. The agar gel in contact with the specimen (approximately $0.5 \times 0.5 \mathrm{~cm}$ ) was cut from the plate, and the bacteria were extracted with $2 \mathrm{~mL}$ of $0.9 \% \mathrm{NaCl}$ solution containing Tween ${ }^{\circledR}$ 20. The bacterial suspensions were spread onto agar plates, and the numbers of colonies were counted and converted to CFU per area of the contacted area $\left(\log _{10} \mathrm{CFU} / \mathrm{cm}^{2}\right)$. The bacterial numbers for the Ag dressings were further converted to " $\log _{10}$ reductions" by subtracting $\log _{10} \mathrm{CFU} / \mathrm{cm}^{2}$ of a dressing containing no antibacterial agents (Aquacel ${ }^{\mathbb{R}}$; ConvaTec Inc., Princeton, NJ, USA). The results were classified as "no activity" ( $\log _{10}$ reduction $<0.5)$, "slight" $\left(\log _{10}\right.$ reduction $\left.=0.5-1\right)$, "significant" $\left(\log _{10}\right.$ reduction $\left.=1-3\right)$, and "strong" $\left(\log _{10}\right.$ reduction $\left.>3\right)$, according to a previous report. ${ }^{20}$

\section{Cytotoxicity evaluation}

The indirect cytotoxicity of the dressings was evaluated using Chinese hamster lung fibroblast (V79) cells, according to the International Organization for Standardization (ISO) 10993-5 standard test method but without determining half maximal inhibitory concentrations $\left(\mathrm{IC}_{50}\right)$. Each dressing (area $5 \times 5 \mathrm{~cm}$ ) was immersed in fresh culture medium for 24 hours. The culture media, containing the water-soluble contents of the dressings, were used for the following cell cultivation tests: The V79 cells were first cultured using MEM containing serum, and then the MEM was replaced with the extraction medium or fresh medium (control). After 7 days of incubation, colonies were fixed with methanol and Giemsa-stained. The stain was extracted from the colonies with $500 \mu \mathrm{L}$ of $50 \%$ ethanol, and the optical density (OD) at $630 \mathrm{~nm}$ was measured with a microplate reader (Sunrise Rainbow; Tecan Group Ltd, Männedorf, Switzerland). Cell growth inhibition was determined by the staining, using the equation:

$$
\begin{aligned}
& \text { Cell growth inhibition }(\%) \\
& =100 \times\left(\mathrm{OD}_{\mathrm{c}}-\mathrm{OD}_{\mathrm{ex}}\right) / \mathrm{OD}_{\mathrm{c}}
\end{aligned}
$$

where $\mathrm{OD}_{\mathrm{ex}}$ and $\mathrm{OD}_{\mathrm{c}}$ were the ODs of the cells cultured in the extraction medium and fresh medium (control), respectively.

The ISO 10993-5 is recommended as a test method for evaluating the biological safety of medical devices in Japan. Among three cell lines (L929, V79, and Balb/3T3) described in the test method, V79 was employed in this study because it forms colonies in the shortest period (6-7 days) among the cell lines. This period is similar to that of the sustained delivery of silver generally exhibited by commercially-available Ag dressings. ${ }^{15}$

\section{Statistics}

The data for the antibacterial evaluation and cytotoxicity evaluations were expressed as mean \pm standard deviation (SD) $(n=3)$ and evaluated by one-way analysis of variance (ANOVA). Statistical significance among groups was then determined by Tukey's test $(P<0.05$ was considered significant). 


\section{Results}

\section{Characteristics of $\mathrm{Ag}^{+}$release}

The amounts of $\mathrm{Ag}^{+}$released were different between $\mathrm{Ag}$ dressings and in the cell culture medium and in ultrapure water (Figure 1). $\mathrm{The}_{\mathrm{Ag}}^{+}$release was indicated as the release amounts per specimen area $\left(\mu \mathrm{g} / \mathrm{cm}^{2}\right)$ and per specimen weight $(\mu \mathrm{g} / \mathrm{g})$. The time-dependent changes of $\mathrm{Ag}^{+}$release were not apparent compared with the significant differences in $\mathrm{Ag}^{+}$release between the samples; the statistical comparison of $\mathrm{Ag}^{+}$release between the samples was performed for the data at an immersion period of 24 hours. The immersion period was identical to the extraction period for the cytotoxicity tests and the contact time for the disc diffusion tests. There were statistically significant differences in $\mathrm{Ag}^{+}$release among Ag dressings (Figure 1).

When the data were indicated as the release amounts per specimen area, an overwhelming release was observed for Mepilex $^{\circledR} \mathrm{Ag}$ in the cell culture medium (Figure 1A). The $\mathrm{Ag}^{+}$release characteristics of Aquacel Ag and Algisite ${ }^{\mathrm{TM}}$ Ag were intermediate among the samples $(0.24 \pm 0.01$ and $0.35 \pm 0.05 \mu \mathrm{g} / \mathrm{cm}^{2}$, respectively). Biohesive Ag and PolyMem ${ }^{\circledR}$ Ag showed very low release $\left(<0.1 \mu \mathrm{g} / \mathrm{cm}^{2}\right)$; however, statistical significance was not observed between Biohesive Ag and Aquacel Ag in the triplicate specimens.

When the $\mathrm{Ag}^{+}$release values were indicated as the release amounts per specimen weight, the release characteristics were divided into two groups: Biohesive Ag and PolyMem Ag had the lower release $(<1.5 \mu \mathrm{g} / \mathrm{g})$, and Aquacel Ag, Algisite Ag, and Mepilex Ag had the higher release ( $>34 \mu \mathrm{g} / \mathrm{g}$ ) (Figure 1B). The weight per area of Mepilex Ag was much higher than that of Aquacel Ag and Algisite Ag (Table 1), and the overwhelming $\mathrm{Ag}^{+}$release of Mepilex Ag per specimen area became similar to those of Aquacel $\mathrm{Ag}$ and Algisite $\mathrm{Ag}$ when the $\mathrm{Ag}^{+}$release was indicated as the release amount per specimen weight.

Figure $1 \mathrm{C}$ shows the $\mathrm{Ag}^{+}$release obtained by the release test in ultrapure water. The released amounts of $\mathrm{Ag}^{+}$were much higher than those in the cell culture medium, for all the samples. In particular, the released amounts of $\mathrm{Ag}^{+}$from Aquacel $\mathrm{Ag}$ and Mepilex Ag in ultrapure water were 24 times and 300 times higher than those in the cell culture medium, respectively. Our preliminary investigations using total silver analysis by inductively coupled plasma optical emission spectroscopy (ICP-OES) supported the very high release of $\mathrm{Ag}^{+}$from Aquacel $\mathrm{Ag}$ and Mepilex Ag in ultrapure water (data not shown).

\section{Antibacterial activity}

Figure 2 shows representative photographs of the agar plates from the disc diffusion tests using the Ag dressings. The width of the inhibition zone is also shown in the figure. Aquacel Ag and Algisite Ag created clear inhibition zones, indicating that sufficient $\mathrm{Ag}^{+}$was released to produce antibacterial activity around the specimens. The other dressings created small or no inhibition zones.
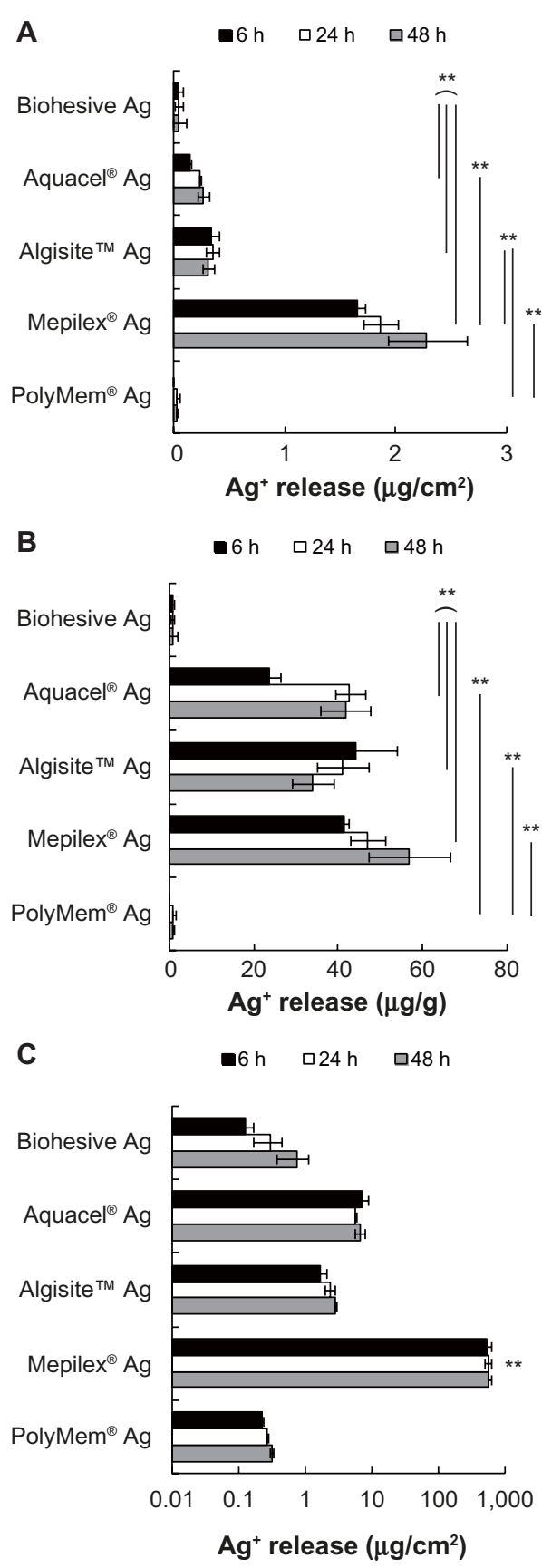

Figure I Release of $\mathrm{Ag}^{+}$from the silver-containing dressings in MEM containing serum or ultrapure water.

Notes: (A) $\mathrm{Ag}^{+}$release per specimen area $\left(\mu \mathrm{g} / \mathrm{cm}^{2}\right)$ in the cell culture medium, (B) $\mathrm{Ag}^{+}$release per specimen weight $(\mu \mathrm{g} / \mathrm{g})$ in cell culture medium, (C) $\mathrm{Ag}^{+}$release per specimen area $\left(\mu \mathrm{g} / \mathrm{cm}^{2}\right)$ in ultrapure water. The data are presented as mean $\pm \operatorname{SD}(n=3)$. The $P$-values obtained by ANOVA were (A) $2 \times 10^{-8}$; (B) $2 \times 10^{-10}$; and (C) $5 \times 10^{-12}$. The vertical bars indicate the statistical differences between the samples $(* * P<0.0 \mathrm{I})$.

Abbreviations: Ag, silver-containing dressing; ANOVA, analysis of variance; MEM, minimum essential medium; SD, standard deviation; $h$, hours. 


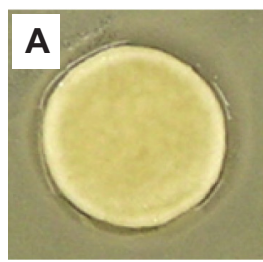

$0.2 \pm 0.1 \mathrm{~mm}$

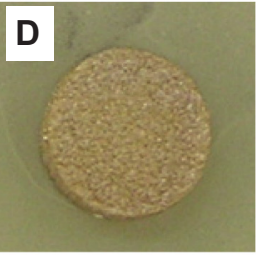

ND

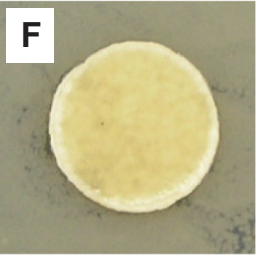

ND

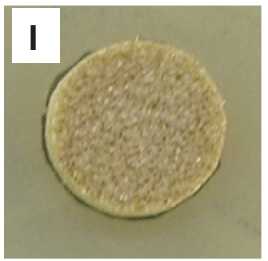

$0.2 \pm 0.1 \mathrm{~mm}$

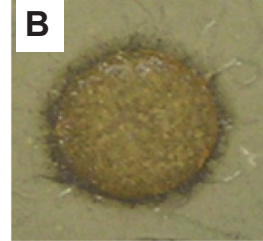

$0.6 \pm 0.2 \mathrm{~mm}$

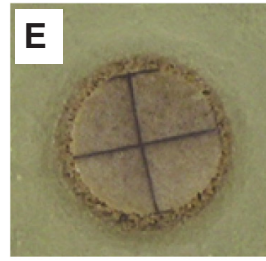

ND

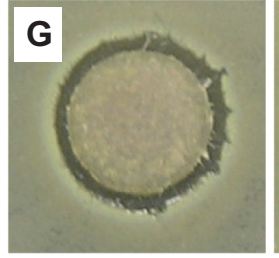

$1.4 \pm 0.1 \mathrm{~mm}$

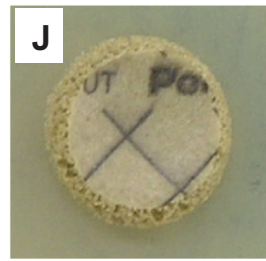

ND

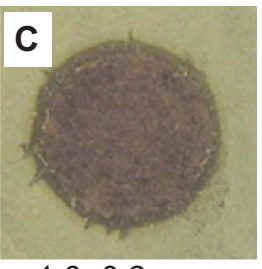

$1.0 \pm 0.2 \mathrm{~mm}$
Figure 2 Representative photographs of agar plates cultured with Staphylococcus aureus (A-E) or Pseudomonas aeruginosa (F-J), following the disc diffusion test with the Ag dressings.

Notes: Biohesive $\mathrm{Ag}(\mathbf{A}$ and $\mathbf{F})$; Aquace ${ }^{\circledR} \mathrm{Ag}(\mathbf{B}$ and $\mathbf{G})$; Algisite ${ }^{\mathrm{TM}} \mathrm{Ag}(\mathbf{C}$ and $\mathbf{H})$; Mepilex ${ }^{\circledast} \mathrm{Ag}$ ( $\mathbf{D}$ and $\left.\mathbf{I}\right)$; and PolyMem ${ }^{\circledast} \mathrm{Ag}(\mathbf{E}$ and $\mathbf{J})$. The width of the inhibition zone (mean \pm standard deviation) $(n=3)$ is shown at the bottom of each picture. ND indicates not detected $(<0.1 \mathrm{~mm})$

The CFU per area and $\log _{10}$ reduction of Ag dressings are presented in Figures 3 and 4. Against gram-positive bacterium S. aureus, there was a significant difference in CFU per area among Ag dressings $\left(P=3 \times 10^{-10}\right)$ (Figure 3). Biohesive Ag, Aquacel Ag, and Algisite Ag showed antibacterial activity classified as "strong," where there was a significant difference in CFU per area between Algisite Ag and Biohesive $\mathrm{Ag}(P<0.01)$. The antibacterial activity of PolyMem Ag was classified as "significant". Mepilex Ag showed the lowest reduction of bacteria; the antibacterial activity was classified as "no activity." A similar tendency was observed in the antibacterial tests against the gram-negative bacterium $P$. aeruginosa, although the order of antibacterial activity of PolyMem Ag and Mepilex Ag was reversed ("no activity" and "significant", respectively), and Biohesive Ag was classified

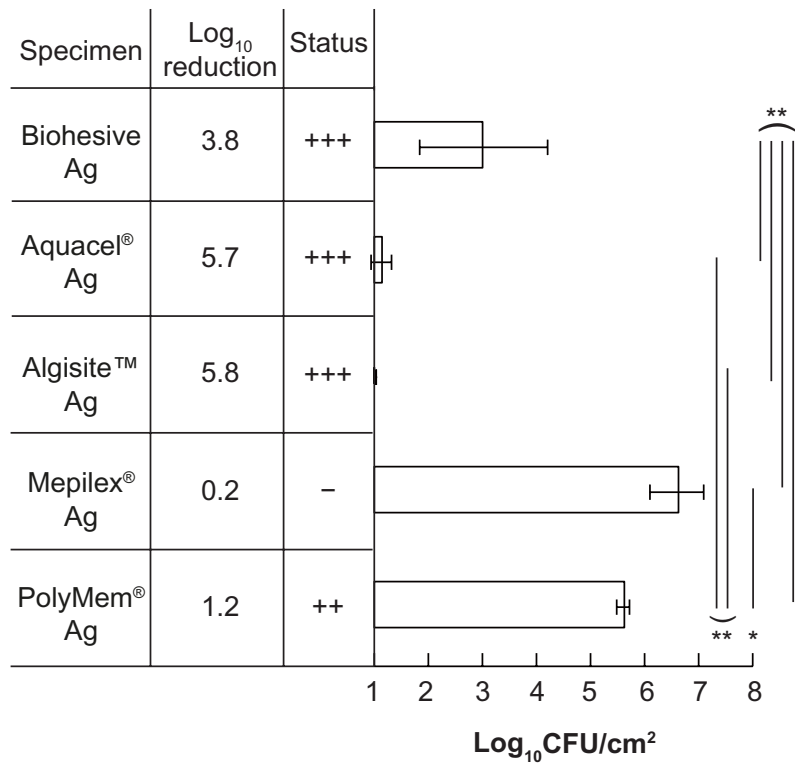

Figure 3 Antibacterial activity of the Ag dressings against Staphylococcus aureus. Notes: The graph indicates CFU per area (mean \pm standard deviation) $(n=3)$ : the bacterial activity in the area where the dressings were in contact with the agar plate was measured by counting the viable bacteria. The initial number of bacterial colonies on the agar plate was set to $3.4 \times 10^{7} \mathrm{CFU}$ per area. The $P$-value obtained by ANOVA was $3 \times 10^{-10}$. The vertical bars indicate the statistical differences between the samples $(* P<0.05$; **P $<0.01)$. ( -$)$ indicates no "antibacterial activity" $\left(\log _{10}\right.$ reduction $\left.<0.5\right)$; $(++)$ indicates "significant antibacterial activity" ( $\log _{10}$ reduction $\left.=\mathrm{I}-3\right)$; and $(+++)$ indicates "strong" $\left(\log _{10}\right.$ reduction $\left.>3\right)$.

Abbreviations: ANOVA, analysis of variance; CFU, colony forming unit.

as "significant" (Figure 4). There was a significant difference in CFU per area among Ag dressings $\left(P=7 \times 10^{-10}\right)$. The statistical differences in CFU per area between every pair of samples are indicated in Figure 4.

\section{Cytotoxicity}

Figure 5 reveals the V79 cell growth inhibition obtained by the cytotoxicity evaluation. There was a significant difference in the cell growth inhibition among Ag dressings $\left(P=2 \times 10^{-17}\right)$. Biohesive Ag exhibited an inhibition of cell growth $(25 \% \pm 19 \%)$ significantly lower than those of the other Ag dressings $(>49 \%)(P<0.01)$. Among the remaining Ag dressings, a statistically significant difference in cell growth inhibition was observed only between Aquacel Ag and Mepilex Ag $(P<0.05)$.

\section{Discussion}

Ag dressing has become a standard choice for conservative wound management, to achieve optimal wound bed preparation by reducing the bioburden. ${ }^{1,2}$ The antibacterial activity of Ag dressings has been well established; however, there is little information on the relationship between the risks (cytotoxicity and resulting delayed wound healing) and benefit (sufficient antibacterial activity), which are determined 


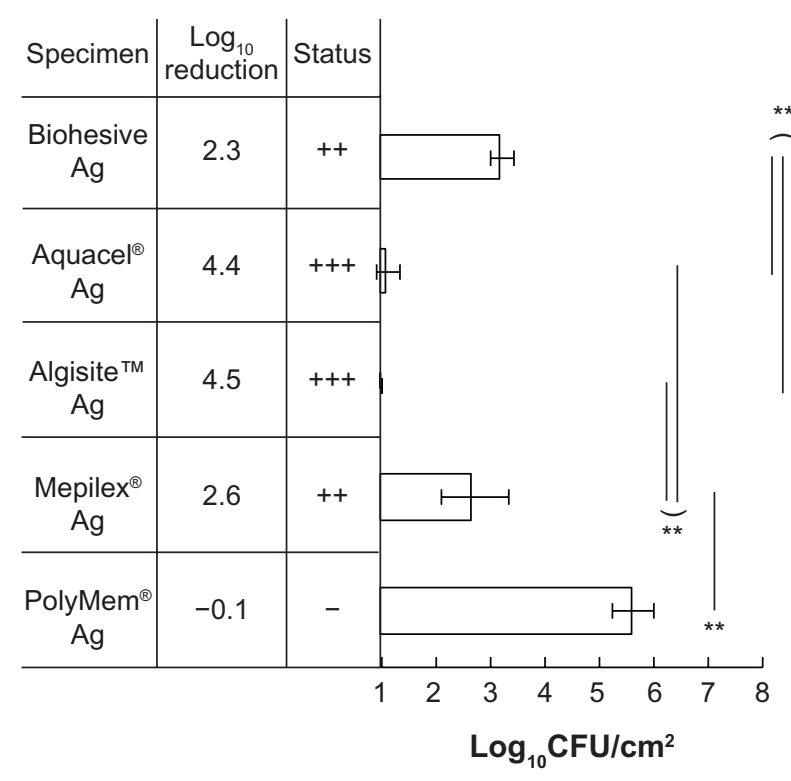

Figure 4 Antibacterial activity of the Ag dressings against Pseudomonas aeruginosa. Notes: The graph indicates CFU per area (mean \pm standard deviation) $(n=3)$ : the bacterial activity in the area where the dressings were in contact with the agar plate was measured by counting the viable bacteria. The initial number of bacterial colonies on the agar plate was set to $5.4 \times 10^{6} \mathrm{CFU}$ per area. The $P$-value obtained by ANOVA was $7 \times 10^{-10}$. The vertical bars indicate the statistical differences between the samples $(* * P<0.01)$. ( $(-)$ indicates "no antibacterial activity" $\left(\log _{10}\right.$ reduction $<0.5)$; $(++)$ indicates "significant antibacterial activity" $\left(\log _{10}\right.$ reduction $\left.=I-3\right)$; and $(+++)$ indicates "strong antibacterial activity" $\left(\log _{10}\right.$ reduction $\left.>3\right)$.

Abbreviations: ANOVA, analysis of variance; CFU, colony forming unit.

by the chemical properties of the silver compounds and the physical properties of the polymeric substrates. This study revealed that the efficacies of $\mathrm{Ag}$ dressings and their adverse effects were different between commercially available $\mathrm{Ag}$ dressings, thus, suggesting that these differences should be considered by the clinicians during wound management.

The most notable finding in this study was the relationship between the antibacterial activity and cytotoxicity of commercially available Ag dressings. Before the study, we had expected that antibacterial activity would increase monotonically as a function of cytotoxicity. In contrast, cytotoxicity was not a monotonic function of antibacterial activity. Furthermore, the functions could not be simply explained by $\mathrm{Ag}^{+}$release. To understand this remarkable relationship, several factors should be considered, including water solubility of the silver compounds, substrate properties, and principles of the test methods.

The higher antibacterial activity and cytotoxic effects of Aquacel Ag and Algisite Ag were simply due to substantial releases of $\mathrm{Ag}^{+}$, as demonstrated in the silver release tests and the disc diffusion tests. These Ag dressings have relatively simple compositions, and it is reasonable that the functions of the dressings only depend on $\mathrm{Ag}^{+}$release. The nonwoven fabric substrates of Aquacel Ag and Algisite Ag

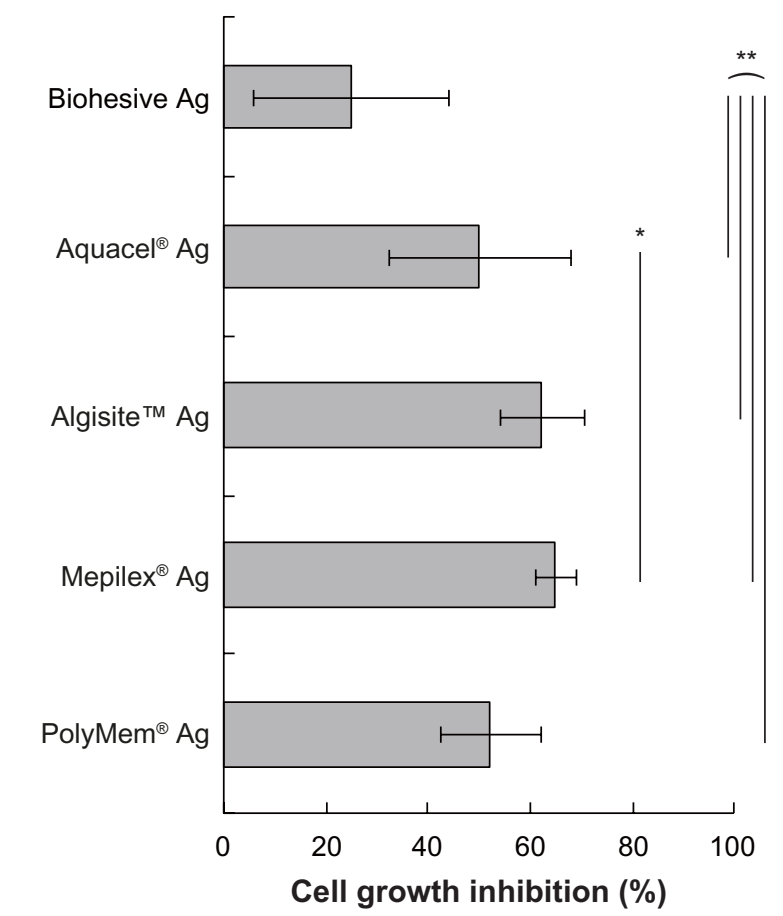

Figure 5 Cell growth inhibition of the $\mathrm{Ag}$ dressings as determined by the indirect cytotoxicity test.

Notes: Each dressing was immersed in fresh culture medium for 24 hours, and the culture media containing the water-soluble contents of the dressings were used for the V79 cell cultivation. Cell growth inhibition was a variable ranging from $0 \%$ (cell growth is equal to that in fresh culture medium) to $100 \%$ (complete cell death). The $P$-value obtained by ANOVA was $2 \times 10^{-17}$. The vertical bars indicate the statistical differences between the samples $(* P<0.05 ; * * P<0.01)$.

Abbreviation: ANOVA, analysis of variance.

are carboxymethylcellulose and alginate, respectively. $\mathrm{Ag}^{+}$ is introduced into the anionic polymer substrates by ion exchange in silver nitrate solution or other silver compound solution. ${ }^{20,27}$ Both the substrates are anionic polymers containing carboxyl groups as ion exchange sites for $\mathrm{Ag}^{+}$, and the release of $\mathrm{Ag}^{+}$occurs easily with the presence of other organic and inorganic ions.

Aquacel Ag and Algisite Ag could each be categorized as a "fast-acting" antibacterial wound dressing based on the findings that substantial amounts of $\mathrm{Ag}^{+}$were rapidly released in 6 hours. However, the high $\mathrm{Ag}^{+}$release is a potential risk factor for histological damage and resulting delayed healing. High cytotoxicity of these Ag dressings has been demonstrated in previous reports. ${ }^{18,20}$ It has been reported that silver was dissociated from Aquacel Ag in contact with body fluids. ${ }^{28}$ The nonwoven fabric substrates show rapid uptake of environmental liquids and diffusion of solutes, accelerating the diffusion of $\mathrm{Ag}^{+}$. Clinicians in wound management take many factors into consideration when choosing Ag dressings: level of exudate production, condition of the wound bed, patient preference, clinical evidence, and cost 
effectiveness. ${ }^{15}$ The relationship between antibacterial and cytotoxic properties could be additional information for clinicians choosing Ag dressings.

Biohesive Ag showed the lowest cytotoxicity among the Ag dressings. The antibacterial activity was lower than those of Aquacel Ag and Algisite Ag but classified as strong or significant. This unique relationship between antibacterial activity and cytotoxicity was probably due to the lower $\mathrm{Ag}^{+}$ release demonstrated by the silver release tests. The extremely low water solubility of $\operatorname{AgSD}(3 \mu \mathrm{M})^{23}$ could contribute to the lower release. Pectin, a cationic hydrocarbon contained in Biohesive Ag (according to the product data), may adsorb $\mathrm{Ag}^{+}$. The dissolution of $\mathrm{AgSD}$ is at equilibrium in solution, by which AgSD is considered to act as a "reservoir" of $\mathrm{Ag}^{+} .{ }^{12}$ This fact characterizes Biohesive Ag as a slow-release Ag dressing.

Despite the low water solubility of AgSD, previous studies have demonstrated the similarity of antibacterial activity between $\mathrm{Ag}^{+}$releasing compounds and AgSD. Carr et $\mathrm{al}^{30}$ reported that the minimum inhibitory effect of $\mathrm{AgSD}$ against $S$. aureus was similar to that of silver as a source of $\mathrm{Ag}^{+}$. In an in vivo study by Chen et al, ${ }^{31}$ similar decreases in bacteria were observed on second-degree burn wounds in response to a dressing containing $\mathrm{Ag}^{+}$and a cream containing $\mathrm{AgSD}$. In several cases, the $\mathrm{Ag}^{+}$release from $\mathrm{AgSD}-$ containing materials may exert strong antibacterial activity in wound sites. In addition, the release characteristics of $\mathrm{Ag}^{+}$ from $\mathrm{AgSD}$ in the human body may be complex. Previous study suggested that the solubility of AgSD increases in the presence of ligands (amino acids and proteins) despite its extremely low solubility in water. ${ }^{31}$ However, we must note that our results and previous findings cannot ensure that $\mathrm{Ag}^{+}$ released from AgSD causes sufficient antibacterial activity for every infected wound. The activity of Biohesive Ag was actually low compared with that of Aquacel Ag and Algisite $\mathrm{Ag}$, suggesting that appropriate selection of Ag dressings are required depending on patients' conditions (in particular, bioburden in the wound site and immunological condition of the patient).

Mepilex Ag, in which a foam substrate was used, showed low antibacterial activity but strong cytotoxicity comparable with that in dressings containing $\mathrm{Ag}^{+}$. The silver release tests ensured that Mepilex Ag showed an extremely high $\mathrm{Ag}^{+}$release, and therefore the strong cytotoxicity can be explained by the $\mathrm{Ag}^{+}$release characteristics. The specimens were soaked in the cell culture medium for the silver release tests and cytotoxicity tests, where silver sulfate contained in the substrate entirely released into the medium by electrolytic dissociation. In the disc diffusion test evaluating antibacterial activity, by contrast, silver existing around the interface between the substrate and the agar gel contributed to the antibacterial activity. In the case of Mepilex Ag, its unique bilayer structure of substrate (silicone foam containing water-soluble silver sulfate as a main layer and a hydrophobic silicone membrane with micropores as a contact layer) did not appear to correspond with the disc diffusion test results. In the interface between the contact layer and agar plate, bacteria cultured on the agar could not contact silver contained in the main layer, resulting in low activity in the antibacterial tests. In contrast to our results, a previous study demonstrated that the silicone foam dressing containing silver had strong antibacterial activity against both $S$. aureus and $P$. aeruginosa. ${ }^{32}$ This contradiction is probably due to differences in water diffusion at the interface between the contact layer and agar plate. It appears that the antibacterial activity of Mepilex Ag depends on the diffusion of body fluid in the interface between a wound site and the dressing.

For PolyMem Ag, the poor antibacterial activity was observed at the same time as strong cytotoxicity. This unique relationship was probably due to the characteristics of nanocrystalline silver (metallic or uncharged form of silver $\left.\left[\mathrm{Ag}^{0}\right]\right)$, regarded as a slowly released silver. ${ }^{12}$ The poor antibacterial activity is supported by a previous report ${ }^{7}$ and can be explained by the lowest $\mathrm{Ag}^{+}$release demonstrated by the silver release tests. The $\mathrm{Ag}^{0}$ form released from nanocrystalline silver is far less rapidly deactivated by chloride or organic matter than the ionic form. ${ }^{33}$ In solution, it exists in a subcrystalline form, less than eight atoms in size, producing $\mathrm{Ag}^{+}$by its equilibrium manner. Furthermore, nanocrystalline silver would be less diffusive in the disc diffusion test, preventing $\mathrm{Ag}^{+}$release outside of the substrate.

The strong cytotoxicity of PolyMem Ag was inconsistent with the lowest $\mathrm{Ag}^{+}$release. Although our data showed no evidence to explain the strong cytotoxicity of PolyMem Ag, we speculated that mammalian cells can uptake the larger size of silver clusters by endocytosis. The test solution for the cytotoxicity test was prepared by soaking the specimen in the cell culture medium, where nanocrystalline silver or its degraded states could be released more than in the disc diffusion test using agar gels as the media. There is no report on the comparison of silver uptake between mammalian cells and bacteria; such comparative study would provide useful information in determining the "risk-benefit" relationship of Ag dressings. PolyMem Ag can be regarded as an Ag dressing that exhibits a unique "risk-benefit" relationship, based on the physicochemical properties of nanocrystalline silver. 
The advantage of our study is that the antibacterial activity and cytotoxicity of Ag dressings were compared with the $\mathrm{Ag}^{+}$release properties in the cell culture medium. Most studies of Ag dressings have employed atomic absorption spectrometry (AAS) as an analytical method for assaying silver release. ${ }^{7,10}$ However, the conventional hot nitric acid treatments used for sample preparation can change $\mathrm{Ag}^{0}$ to $\mathrm{Ag}^{+}$. It is widely accepted that $\mathrm{Ag}^{+}$plays a crucial role in the biological functions of silver. AAS analyses would detect, not only bioactive $\mathrm{Ag}^{+}$but also, less bioactive $\mathrm{Ag}^{0}$. We employed the silver assay reaction to determine " $\mathrm{Ag}^{+}$" concentrations in the releasing media. Furthermore, we demonstrated that silver release tests for Ag dressings in ultrapure water resulted in excessive release of $\mathrm{Ag}^{+}$because of the absence of organic and inorganic ions. The behavior of $\mathrm{Ag}^{+}$in the cell culture medium is complex and involves the formation of silver complexes with amino acids, proteins, and chloride ions. ${ }^{29}$ It is apparent that the silver release test should be conducted in releasing media in which the components mimic those of body fluids.

One limitation of this study is that antibacterial activity was evaluated only by the disc diffusion test, in which less diffusive forms, such as nanocrystalline silver, are restricted by rigid agar gels and in which antibacterial activity exhibited at the interior of substrates is not detected. Our results suggest that the antibacterial activity of $\mathrm{Ag}$ dressings in the disc diffusion test was underestimated, depending on the structures and properties of substrates. In future studies, different antibacterial tests and in vivo wound healing tests should be conducted to obtain further information on the relationship between antibacterial activity and cytotoxicity.

We must also note limitation of the cytotoxicity tests attributed to the lack of $\mathrm{IC}_{50}$ determination. The result of cell growth inhibitions without $\mathrm{IC}_{50}$ determination is useful only for comparing the strength of cytotoxicity among $\mathrm{Ag}$ dressings. Human fibroblasts were a possible candidate for the test cells, but we placed higher importance on the use of established and recommended cell lines (V79) in the standard test method, to obtain more reliable and reproducible data for cytotoxicity.

\section{Conclusion}

Five commercially available Ag dressings (Biohesive Ag, Aquacel Ag, Algisite Ag, Mepilex Ag, and PolyMem Ag) were investigated in vitro for a parallel evaluation of their antibacterial activity and cytotoxicity. These properties were different between the Ag dressings, and we found that cytotoxicity was not a monotonic function of antibacterial activity. Furthermore, the antibacterial activity and cytotoxicity could not be simply explained by $\mathrm{Ag}^{+}$release properties, suggesting that Ag dressings have different characteristics depending, not only on $\mathrm{Ag}^{+}$release but also, on other factors, such as the species of silver released and substrate properties. It can be concluded that the risks and benefits differ among $\mathrm{Ag}$ dressings and that clinicians should take these differences into consideration during wound management.

\section{Acknowledgments}

The authors thank Dr H Hayashi for his helpful advice on silver analyses by ICP-OES, and Enago (http:Ilwww.enago.jp) for the English language review.

\section{Author contributions}

All authors substantially contributed to the conception and design of this study, acquisition and analysis of data, interpretation of data, drafting the article or revising it critically for important intellectual content, and final approval of the version to be submitted. Furthermore, all authors agreed that they are accountable for all aspects of the work, ensuring that questions related to the accuracy or integrity of any part of the work were appropriately investigated and resolved.

\section{Disclosure}

The authors report no conflicts of interest in this work.

\section{References}

1. Leaper DJ, Schultz G, Carville K, Fletcher J, Swanson T, Drake R. Extending the TIME concept: what have we learned in the past 10 years?(*). Int Wound J. 2012;9 Suppl 2:S1-S19.

2. Sibbald RG, Goodman L, Woo KY, et al. Special considerations in wound bed preparation 2011: an update C. Adv Skin Wound Care. 2011;24(9):415-436; quiz 437.

3. Grant GT, Morris ER, Rees DA, Smith PJC, Thom D. Biological interactions between polysaccharides and divalent cations: The egg box model. FEBS Lett. 1973;32(1):195-198.

4. Sandri G, Bonferoni MC, D'Autilia F, et al. Wound dressings based on silver sulfadiazine solid lipid nanoparticles for tissue repairing. Eur J Pharm Biopharm. 2013;84(1):84-90.

5. White RJ, Cutting K, Kingsley A. Topical antimicrobials in the control of wound bioburden. Ostomy Wound Manage. 2006;52(8):26-58.

6. Ip M, Lui SL, Poon VK, Lung I, Burd A. Antimicrobial activities of silver dressings: an in vitro comparison. J Med Microbiol. 2006;55(Pt 1):59-63.

7. Parsons D, Bowler PG, Myles V, Jones S. Silver antimicrobial dressing in wound management: A comparison of antibacterial, physical, and chemical characteristics. Wounds. 2005;17(8):222-232.

8. Basterzi Y, Ersoz G, Sarac G, Sari A, Demirkan F. In-vitro comparison of antimicrobial efficacy of various wound dressing materials. Wounds. 2010;22(7):165-169.

9. Percival SL, Bowler PG, Russell D. Bacterial resistance to silver in wound care. J Hosp Infect. 2005;60(1):1-7. 
10. Cavanagh MH, Burrell RE, Nadworny PL. Evaluating antimicrobial efficacy of new commercially available silver dressings. Int Wound J. 2010;7(5):394-405.

11. Liau SY, Read DC, Pugh WJ, Furr JR, Russell AD. Interaction of silver nitrate with readily identifiable groups: relationship to the antibacterial action of silver ions. Lett Appl Microbiol. 1997;25(4):279-283.

12. Atiyeh BS, Costagliola M, Hayek SN, Dibo SA. Effect of silver on burn wound infection control and healing: review of the literature. Burns. 2007;33(2):139-148.

13. Jørgensen B, Price P, Andersen KE, et al. The silver-releasing foam dressing, Contreet Foam, promotes faster healing of critically colonised venous leg ulcers: a randomised, controlled trial. Int Wound J. 2005;2(1):64-73.

14. Muangman P, Pundee C, Opasanon S, Muangman S. A prospective, randomized trial of silver containing hydrofiber dressing versus $1 \%$ silver sulfadiazine for the treatment of partial thickness burns. Int Wound J. 2010;7(4):271-276.

15. International consensus. Appropriate Use of Silver Dressings in Wounds. An Expert Working Group Consensus. London: Wounds International; 2012. Available from: http:/www.woundsinternational.com/pdf/ content_10381.pdf. Accessed January 24, 2014.

16. Hollinger MA. Toxicological aspects of topical silver pharmaceuticals. Crit Rev Toxicol. 1996;26(3):255-260.

17. Poon VK, Burd A. In vitro cytotoxity of silver: implication for clinical wound care. Burns. 2004;30(2):140-147.

18. Zou SB, Yoon WY, Han SK, Jeong SH, Cui ZJ, Kim WK. Cytotoxicity of silver dressings on diabetic fibroblasts. Int Wound J. 2013;10(3):306-312.

19. Fredriksson C, Kratz G, Huss F. Accumulation of silver and delayed re-epithelialization in normal human skin: An ex-vivo study of different silver dressings. Wounds. 2009;21(5):116-123.

20. Wiegand C, Heinze T, Hipler UC. Comparative in vitro study on cytotoxicity, antimicrobial activity, and binding capacity for pathophysiological factors in chronic wounds of alginate and silver-containing alginate. Wound Repair Regen. 2009;17(4):511-521.

21. Burd A, Kwok CH, Hung SC, et al. A comparative study of the cytotoxicity of silver-based dressings in monolayer cell, tissue explant, and animal models. Wound Repair Regen. 2007;15(1):94-104.
22. Wilkinson LJ, White RJ, Chipman JK. Silver and nanoparticles of silver in wound dressings: a review of efficacy and safety. $J$ Wound Care. 2011;20(11):543-549.

23. Nesbitt RU, Sandmann BJ. Solubility studies of silver sulfonamides. J Pharm Sci. 1978;67(7):1012-1017.

24. Ohshita K, Wada H, Nakasawa G. Spectrophotometric determination of silver with 4-(3,5-dibromo-2-pyridylazo)-N,N-diethylaniline in the presence of sodium dodecylsulfate. Analytica Chimica Acta. 1986;182:157-162.

25. Japanese Industrial Standard Community. Testing for antimicrobial activity and efficacy on the textile products (JIS L 1902). Tokyo: Japanese Standard Association; 2008. Available from: http:/www. webstore.jsa.or.jp/webstore/Com/FlowControl.jsp?lang=en\&bunsyold= JIS $+\mathrm{L}+1902 \% 3$ A2008\&dantaiCd $=$ JIS\&status $=1 \&$ pageNo $=9$. Accessed December 15, 2014.

26. Konya C, Sanada H, Sugama J, et al. Skin debris and micro-organisms on the periwound skin of pressure ulcers and the influence of periwound cleansing on microbial flora. Ostomy Wound Manage. 2005;51(1):50-59.

27. Burrell RE. A scientific perspective on the use of topical silver preparations. Ostomy Wound Manage. 2003;49(5A Suppl):S19-S24.

28. Ovington LG. The truth about silver. Ostomy Wound Manage. 2004;50(9A Suppl):1S-10S.

29. Tsipouras N, Rix CJ, Brady PH. Solubility of silver sulfadiazine in physiological media and relevance to treatment of thermal burns with silver sulfadiazine cream. Clin Chem. 1995;41(1):87-91.

30. Carr HS, Wlodkowski TJ, Rosenkranz HS. Silver sulfadiazine: in vitro antibacterial activity. Antimicrob Agents Chemother. 1973;4(5):585-587.

31. Chen J, Han CM, Lin XW, Tang ZJ, Su SJ. [Effect of silver nanoparticle dressing on second degree burn wound]. Zhonghua Wai Ke Za Zhi. 2006;44(1):50-52. Chinese.

32. Barrett S. Mepilex Ag: an antimicrobial, absorbent foam dressing with Safetac technology. Br J Nurs. 2009;18(20):S28, S30-S36.

33. Dunn K, Edwards-Jones V. The role of Acticoat with nanocrystalline silver in the management of burns. Burns. 2004;30 Suppl 1:S1-S9.
Chronic Wound Care Management and Research

\section{Publish your work in this journal}

Chronic Wound Care Management and Research is an international, peer reviewed, open access, online journal publishing original research, reviews, editorials, and commentaries on the causes and management of chronic wounds and the major issues related to chronic wound management. Topics also include chronic wounds as comorbidities to other

\section{Dovepress}

conditions, patient adherence to therapy, and the economic burden of chronic wounds. The manuscript management system is completely online and includes a very quick and fair peer review system, which is all easy to use. Visit http://www.dovepress.com/testimonials.php to read real quotes from published authors. 